\title{
Factores determinantes del acceso a la educación superior para estudiantes de zonas rurales, Huaraz - Perú
}

\author{
Fiorella Rosario del Castillo Palacios \\ fdelcastillo@unasam.edu.pe \\ Betsabe Magali Yañac Huerta \\ byanach@unasam.edu.pe \\ Gloria Beatriz Castro Palma \\ gcastrop@unasam.edu.pe
}

Universidad Nacional Santiago Antúnez de Mayolo

Huaraz-Perú

\section{RESUMEN}

La presente investigación tiene como objetivo principal identificar los factores determinantes del acceso a la educación superior de estudiantes de zonas rurales, Huaraz; en consecuencia, los objetivos específicos están orientados a precisar los factores personal, familiar y contextual. La muestra aleatoria lo conforman los estudiantes de zona rural de las instituciones educativas pertenecientes a la Ugel Huaraz en un total de 134. El estudio es descriptivo simple, se utilizó la técnica de la encuesta y el instrumento aplicado fue el cuestionario conformado por veinte preguntas. Los resultados obtenidos demuestran que el factor personal define en un $76.1 \%$, de los estudiantes su rendimiento académico y aspiración educativa para alcanzar los estudios superiores. El factor familiar obtuvo el $44 \%$, ello indica que el respaldo de la familia, en especial de los padres, es determinante en el acceso a la educación superior. Por último, en la dimensión contextual $52.2 \%$ considera que el acceso geográfico y cultural concretarían su oportunidad de acceder la educación superior.

Palabras clave: factor personal; factor familiar, factor contextual, educación superior. 


\title{
Determinants of access to higher education for students from rural areas, Huaraz - Peru
}

\begin{abstract}
The present research aims to identify the key determinants of the access to the higher education of students of rural zones in Huaraz. Consequently, the specific objectives are aimed at identifying the personal, family, and contextual factors. The random sample is made up of 134 rural students of the educational institutions belonging to the Ugel Huaraz. The study is simple descriptive. The technique was the survey and the applied instrument was the questionnaire, having twenty questions. In general, the findings show that $53.0 \%$ of students consider personal, family and contextual factors as determinants in order to access to higher education. Particularly, the personal factor got $76.1 \%$, in which students analyze their academic performance and educational aspiration to reach higher education. In the family factor, $44 \%$ indicate that support from the family, especially parents, is also determinant in access to higher education. Finally, in the contextual dimension, $52.2 \%$ consider that geographical and cultural access would provide them with the opportunity to access higher education.
\end{abstract}

Keywords: personal factor; family factor; contextual factor; higher education.

Artículo recibido: 05 octubre. 2021 Aceptado para publicación: 02 noviembre 2021 Correspondencia: fdelcastillo@unasam.edu.pe Conflictos de Interés: Ninguna que declarar 


\section{INTRODUCCIÓN}

En el presente artículo de investigación se aborda los factores determinantes del acceso a la educación superior para estudiantes de zonas rurales, Huaraz y el objetivo principal precisamente es identificar los factores determinantes del acceso a estudios superiores. Como marco para entender cuál es la realidad que contextualiza este problema de investigación iniciamos el análisis en lo internacional, tenemos el caso de México y Argentina a través de un estudio realizado por Formichella, Krüger y Rojas (2006), quienes identificaron en su investigación que los más afectados frente a un cambio en las políticas económicas son las personas de escasos recursos, puesto que no han cambiado los valores de repitencia, abandono escolar; y que la influencia del ambiente y la economía de las familias sigue sin prosperar. En un análisis hecho por Guzmán y Serrano (2010), en la ciudad de México, referido al concurso de selección para el ingreso a la Universidad Nacional Autónoma de México concluye que existe un desfase entre el nivel secundaria y superior; por incidencia en los resultados dado que los estudiantes de instituciones privadas son favorecidos, siendo el resultado del acceso a la educación superior inequitativo. Asimismo, en el estudio realizado por Gessaghi y Llinas (2005) a través del centro de implementación de políticas públicas para la equidad y el crecimiento en Argentina indica que la población de escasos recursos a veces no alcanza no solo el nivel superior, sino también la educación secundaria.

En el ámbito nacional, Díaz (2014) sostiene que en el Perú también encontramos serias diferencias en cuanto a la calidad educativa. Sostiene que, la diferencia en la preparación académica que ofrecen los centros educativos particulares de zonas urbanas es muy distante a la que se encuentra en zonas rurales. Por otro lado, los estudios realizados por la OECD (2016) afirman que las estadísticas de estudiantes de educación secundaria se han incrementado de $32.1 \%$ a $40.5 \%$; no obstante, la superior creció mínimamente de $15.1 \%$ a un $19.2 \%$, cuya principal limitante es el ingreso económico de las familias.

En el ámbito local, la información obtenida por la Oficina General de Estudios de la Universidad Nacional Santiago Antúnez de Mayolo es que en el examen de admisión 2017-1 solo ingresaron 85 estudiantes de comunidades campesinas, pero no todas ubicadas en zona rural y pertenecientes a las demás provincias del departamento de Áncash. 
Algunos trabajos previos que respaldan esta investigación: Said (2011), informe Rol de los progenitores en el acceso a la educación superior respaldado por la Universidad Nacional del Nordeste de Argentina. Entre las conclusiones afirma que los factores ambientales transmiten cultura y carácter de los estudiantes en relación con su rendimiento académico. Lo mismo en la preparación y empleo de los padres deciden el acceso a la educación superior y desempeño estudiantil en la institución.

Pérez, Ramos, Adiego y Cerno (2012) título Principales determinantes del acceso a la educación superior en España: ¿Existen diferencias antes y después de la crisis? En cuanto a las conclusiones sostiene que la demanda en educación superior entre los años de estudio 2006 y 2010 se redujo en padres con carreras técnicas y madres en ocupaciones no profesionales, pero se incrementa la demanda cuando ambos trabajan y son profesionales.

Almánzar (2013) titulado, Determinantes socioeconómicos de la demanda de educación superior en República Dominicana. Los factores que condicionan el acceso a la educación superior son sociales, económicas y personales. Otro es la ubicación de los usuarios, los que viven en zona urbana tienen mayor acceso a los de rural.

En el plano nacional, Guerrero (2013) investigación titulada ¿Cómo afectan los factores individuales y escolares en la decisión de los jóvenes de postular a la educación superior - Lima? Entre las conclusiones se resalta que la condición social y el nivel socioeconómico de las familias constituyen las principales variables para acceder a la educación superior. Asimismo, influye el apoyo que la institución educativa en el desarrollo académico y lo inclina a postular a la educación superior.

Moreno (2013), Factores socio-económicos-culturales y su influencia en la demanda de una carrera universitaria en el departamento de La Libertad. Principales conclusiones es que la familia y el nivel socioeconómico influyen en la demanda de una carrera universitaria. Se refuerza a la familia como orientadora y determinante en la elección de la carrera, sea por las condiciones socioeconómicas y la vocación profesional.

León y Sugimaru (2013) Entre el estudio y el trabajo, las decisiones de los jóvenes peruanos después de concluir la educación básica regular. Entre las principales conclusiones están que 2 de cada 5 estudiantes tiene que trabajar y solo 1 de 5 estudia superior por los altos costos. Los estudiantes de zona rural son los que menos favorecidos. Asimismo, influye como determinante la carencia del apoyo familiar y del contexto 
educativo.

En el plano local no encontramos trabajos relacionados a la investigación; sin embargo, se conoce que existen convenios entre la Universidad Nacional Santiago Antúnez de Mayolo y las comunidades campesinas. Los beneficiarios son los hijos de comuneros que obtuvieron del 1ro al 5to puesto al terminar la educación secundaria, según Resolución de Consejo Universitario No 090-2017-UNASAM, de fecha de febrero de 2017.

Los factores determinantes son condiciones que el estudiante de educación secundaria deberá enfrentar para alcanzar la educación superior (Sarmiento, 2006). De lo expuesto anteriormente se consolidaron tres dimensiones de la variable como son factor personal, factor familiar y factor contextual y la teoría nos servirá para entenderlos. En el primer factor, lo constituyen todos los aspectos individuales que van a condicionar el logro del ingreso a la educación superior para conseguir este propósito el estudiante recibió durante once años una formación básica integral que va a determinar entre otros su rendimiento académico y sus aspiraciones educativas. En segundo lugar, el familiar, para las familias el hecho de enviar a un hijo a estudiar educación superior significa una preocupación porque sus gastos se ven incrementados, ya que no solo es dinero sino sacrificio, angustias y limitaciones (López, Espinoza, Rojo y Rojas, 2012). Asimismo, el rol de la familia desde el punto de vista social ejerce una gran influencia, para Benavides, Olivera y Mena (2006) existen dos teorías para analizar el rol que las familias cumplen en el proceso educativo de sus hijos. Uno de ellos es el capital humano y otro, lo cultural. Dos posiciones que sostienen que las personas necesitan estar capacitadas en el nivel cognitivo, cultural y educativo para mejorar su desempeño profesional. Por último, el factor contextual, pues la educación se desarrolla dentro de la sociedad influye y condiciona el actuar. Es por ello que el contexto determina muchas veces en qué condiciones se puede ejecutar el anhelo de estudiar educación superior. Dentro de este factor contextual encontramos tres indicadores: acceso geográfico, oferta educativa y acceso cultural.

La hipótesis de investigación que se planteó es: Los factores personal, familiar y contextual son determinantes del acceso a la educación superior para estudiantes de zonas rurales en Huaraz.

La metodología empleada corresponde al diseño de investigación descriptivo simple, pues busca y recoge información con el objeto de estudio. La población lo conforma los 
estudiantes de quinto año de educación secundaria en un número de 225, pertenecientes a dieciocho instituciones educativas de zonas rurales en Huaraz. Para la muestra se determinó que sea un muestreo aleatorio estratificado con afijación proporcional constituyendo un total de 134 estudiantes. Asimismo, la técnica utilizada fue la encuesta, pues recopila datos de una cantidad significativa de personas y se accede científicamente la opinión de estas (Del Cid, Méndez y Sandoval, 2011). En cuanto al instrumento aplicado fue el cuestionario, permite recoger información sobre sucesos, puntos de vista, anhelos de manera organizada. Está dividido en tres dimensiones con veinte preguntas valoradas con la escala de Likert.

\section{RESULTADOS}

Se detallan los resultados obtenidos de la variable general y se describen los resultados de cada uno de los factores.

\section{Tabla 1}

Distribución de frecuencias de los factores determinantes del acceso a la educación superior para estudiantes de zonas rurales, Huaraz 2017

\begin{tabular}{lcc}
\hline \multicolumn{1}{c}{ Acceso a la educación superior } & N & \% \\
\hline Determinante & 18 & 13,4 \\
Medianamente determinante & 45 & 33,6 \\
Determinante & 71 & 53,0 \\
Total & $\mathbf{1 3 4}$ & $\mathbf{1 0 0 , 0}$ \\
\hline \multicolumn{2}{r}{ Fuente. Elaboración propia en base a los resultados de la encuesta }
\end{tabular}

En la tabla 1 se presenta la variable acceso a la educación superior para estudiantes de zonas rurales de las instituciones educativas pertenecientes a la Ugel-Huaraz; en la cual se observa que el $53.0 \%$ del total de estudiantes manifiestan que los factores personal, familiar y contextual, en general, son determinantes del acceso a la educación superior. Es así que la prueba de hipótesis general para esta parte de la investigación se cumple en un $53.0 \%$.

Se observó que el $76.1 \%$ de estudiantes considera que el factor personal es determinante del acceso a la educación superior para estudiantes de zonas rurales y se concluye que el factor en la mayoría de los estudiantes está considerado como determinante del acceso a la educación superior para estudiantes de zonas rurales, Huaraz 2017.Asimismo se 
cumple la hipótesis específica 1 en la cual el factor personal sí es determinante para el acceso a la educación superior.

El $44.0 \%$ de estudiantes manifestó que el factor familiar es determinante del acceso a la educación superior en zonas rurales en la ciudad de Huaraz; por tal razón, la hipótesis de investigación 2 se cumple afirmando que el factor familiar sí es determinante para el acceso a la educación superior.

El 52.2\% de estudiantes manifestaron que el factor contextual del acceso a la educación superior para estudiantes de zonas rurales en la ciudad de Huaraz, es determinante del acceso a la educación superior. Por lo tanto, la hipótesis de investigación 3 se cumple afirmando que el factor contextual sí es determinante del acceso a la educación superior.

\section{CONCLUSIONES}

Como conclusión general de la presente investigación, un $53.0 \%$ de estudiantes de instituciones educativas de zonas rurales de la Ugel-Huaraz afirma que los factores personal, familiar y contextual son determinantes del acceso a la educación superior. Resultado que se contrasta con la comprobación de la hipótesis de investigación; finalmente, la hipótesis nula queda descartada.

El factor personal es determinante en un $76.1 \%$ del acceso a la educación superior. En los resultados del estadístico el valor de la chi cuadrada que es 117.107, está en la región de rechazo de la Hipótesis nula (Ho), es decir el factor personal está asociado al acceso de la educación superior para estudiantes de zonas rurales de Huaraz; se puede afirmar que el este factor es determinante para el acceso a la educación superior para estudiantes de zonas rurales de Huaraz a una confianza de 95\% y se cumple la hipótesis de investigación especifica 1.

El factor familiar es determinante en un $44 \%$ del acceso a la educación superior. En los resultados del estadístico el valor de la chi cuadrada que es 162.267, está en la región de rechazo de la Hipótesis nula (Ho), es decir el factor familiar está asociado al acceso a la educación superior para estudiantes de zonas rurales de Huaraz; por lo que, se puede afirmar que este factor es determinante para el acceso a la educación superior para estudiantes de zonas rurales de Huaraz a una confianza de $95 \%$ y se cumple la hipótesis de investigación especifica 2.

El factor contextual es determinante en un 52.2\% del acceso a la educación superior. En los resultados del estadístico el valor de la chi cuadrada que es 179.938, está en la región 
de rechazo de la Hipótesis nula (Ho), es decir que este factor está asociado al acceso a la educación superior para estudiantes de zonas rurales de Huaraz; por lo que se concluye que el factor contextual es determinante para el acceso a la educación superior para estudiantes de zonas rurales de Huaraz a una confianza de $95 \%$ y se cumple la hipótesis de investigación especifica.

\section{REFERENCIAS BIBLIOGRAFICAS}

Almánzar Guzmán, P. (2013) Determinantes socioeconómicos de la demanda de educación superior en República Dominicana (tesis de maestría). Universidad Católica de Santo Domingo. 75 pp. Recuperado de: http://empirica.do/download/determinantes-socioeconomicos-de-la-demanda-deeducacion-superior-en-republica-dominicana

Benavides, M.; Olivera, Inés y Mena, Magrith (2006). De papás y mamás a hijos e hijas: las aspiraciones sobre el futuro y el rol de las familias en las actividades escolares en el Perú rural . En: GRADE, Grupo de Análisis para el Desarrollo. Los desafíos de la escolaridad en el Perú : estudios sobre los procesos pedagógicos, los saberes previos y el rol de las familias. (págs. 156 - 214). Perú: GRADE, Grupo de análisis para el desarrollo.

Del Cid, A.; Méndez, R. y Sandoval, Franco. (2011). Investigación, fundamentos y Metodología. Segunda edición.México. Recuperado de: https://josedominguezblog.files.wordpress.com/2015/06/investigacionfundamentos-y-metodologia.pdf.

Díaz, Juan José. (2008). Educación superior: tendencias de la demanda y oferta. En: GRADE, Grupo de análisis para el desarrollo. Análisis de programas, proceso y resultados educativos en el Perú: contribuciones empíricas para el debate. Recuperado de: http://bibliotecavirtual.clacso.org.ar/Perugrade/20100405042637/analisis-2.pdf

Formichella, M.; Krüger, N y Rojas, M. (2006). Procesos de descentralización educativa y sus resultados: el caso de México y Argentina. Argentina. Recuperado de: http://www.redalyc.org/pdf/510/51015096005.pdf.

Guerrero, G. (2013). Cómo afectan los factores individuales y escolares en la decisión de los jóvenes de postular a la educación superior. Lima. Recuperado de: http://www.grade.org.pe/publicaciones/1184-como-afectan-los-factores- 
individuales-y-escolares-la-decision-de-los-jovenes-de-postular-a-educacionsuperior-un-estudio-longitudinal-en-lima-peru/

Guessaghi, V. y Llinas (2005). Democratizar el acceso a la educación superior. Argentina. Recuperado de: https://docgo.org/democratizar-el-acceso-a-la-educacionsuperior-1

León, J. y Sugimaru, C. (2013). Entre el estudio y el trabajo, las decisiones de los jóvenes peruanos después de concluir la educación básica regular. Lima. Recuperado de: http://www.grade.org.pe/upload/publicaciones/archivo/download/pubs/AI11.pdf.

López de la Madrid, M.; Espinoza de los Monteros, A. ; Rojo Morales, D. y Rojas García, Alberto. (2012). Disposición y apoyo de los padres de familia a la educación superior. Revista Científica Nova Scientia, 148-164. Recuperado de: http://www.redalyc.org/articulo.oa?id=203324394009

OECD, C. d. (2016). Avanzando hacia una mejor educación para Perú. Lima.

Pérez Esparrels, C; Ramos M.; Adiego, M.; y Cerno, L. (2012). Principales determinantes del acceso a la educación superior en España: ¿Existen diferencias antes y después de la crisis? España. Recuperado de: http://2013.economicsofeducation.com/user/pdfsesiones/176.pdf.

Moreno Rodríguez, R. (2013). Factores socio-económico-culturales y su influencia en la demanda de una carrera universitaria en el departamento de La Libertad. Posgrado Universidad Nacional de Trujillo.

Sarmiento, M. (2006). Rendimiento Académico. Obtenido de http://www.dspace.espol.edu.ec/bitstream/123456789/5713/8capitulo\%20011.do c. 